\title{
Higher Energy States in the CO Dimer: Millimeter-Wave Spectra and Rovibrational Calculations $^{\dagger}$
}

\author{
Leonid A. Surin, Dmitri N. Fourzikov, Thomas F. Giesen, Stephan Schlemmer, and \\ Gisbert Winnewisser
}

I. Physikalisches Institut, Universität zu Köln, Zülpicher Strasse 77, 50937 Cologne, Germany

\author{
Victor A. Panfilov and Boris S. Dumesh \\ Institute of Spectroscopy, Russian Academy of Sciences, 142190 Troitsk, Moscow Region, Russia
}

\author{
Gé W. M. Vissers \\ Department of Chemistry, The Ohio State University, Columbus, Ohio 43210
}

\begin{abstract}
Ad van der Avoird*
Theoretical Chemistry, Institute for Molecules and Materials, Radboud University Nijmegen, Toernooiveld 1, 6525 ED Nijmegen, The Netherlands
\end{abstract}

Received: June 5, 2007; In Final Form: July 10, 2007

\begin{abstract}
New extensive millimeter-wave measurements of the ${ }^{12} \mathrm{C}^{16} \mathrm{O}$ dimer have been made, and more than 300 new spectral transitions have been observed in the frequency range $81-135 \mathrm{GHz}$. A joint analysis of these and previous millimeter-wave data yielded the precise location of 33 new energy levels of $A^{+}$symmetry and 20 levels of $A^{-}$symmetry. These energy levels are located at $8-18 \mathrm{~cm}^{-1}$ above the zero-point level. Some of them belong to already known stacks, and others make up 9 new stacks of the dimer. Newly determined stacks have $K=0,1$, and, for the first time, 2 , where $K$ is the projection of the total angular momentum on the intermolecular axis. The energy levels from accompanying rovibrational calculations with the use of a recently developed hybrid CCSD(T)/DFT-SAPT potential are in very good agreement with experiment. Analysis of the calculated wave functions revealed that two new stacks of $A^{+}$symmetry with $K=2$ correspond to overall rotation of the dimer while the other newly observed stacks belong to the geared bend overtone modes. The ground vibrational states of the two "isomers" found are more or less localized at the two minima in the potential surface, whereas all the geared bend excited states show a considerable amount of delocalization.
\end{abstract}

\section{Introduction}

The carbon monoxide dimer is one of the most mysterious examples of weakly bound molecular complexes, which has resisted many attempts in the past to unravel its properties. After the pioneering but inconclusive report on the microwave study of $(\mathrm{CO})_{2}$ by Klemperer's group in $1979,{ }^{1}$ various research groups tried to approach this problem experimentally ${ }^{2,3}$ and theoretically, ${ }^{4-11}$ but these efforts failed or were of little help in deriving reliable line assignments because of considerable nonrigidity of the complex.

During the last years significant progress has finally been achieved toward securing reliable experimental data in the IR and millimeter-wave regions, thereby establishing for the first time an understanding of the rovibrational and rotational spectra of the CO dimer ${ }^{12-18}$ and its isotopologues. ${ }^{13,20-24}$ There has also been parallel progress in accurate ab initio calculations of a reliable potential energy surface combined with calculations of the rovibrational states of the dimer. ${ }^{25,26}$ The experimental results for $\left({ }^{12} \mathrm{C}^{16} \mathrm{O}\right)_{2}$ are summarized in ref 18 and for the

† Part of the "Giacinto Scoles Festschrift".

* Corresponding author. E-mail: A.vanderAvoird@theochem.ru.nl. $\left({ }^{13} \mathrm{C}^{16} \mathrm{O}\right)_{2}$ and $\left({ }^{12} \mathrm{C}^{18} \mathrm{O}\right)_{2}$ isotopologues in ref 24 . The last paper gives also an extensive description of the current understanding of the structure and dynamics of the $\mathrm{CO}$ dimer based on a recently developed hybrid potential from ab initio coupled cluster $[\mathrm{CCSD}(\mathrm{T})]$ and symmetry-adapted perturbation theory (DFT-SAPT) calculations. ${ }^{25,26}$ Here we briefly summarize the main features of the energy level pattern of the CO dimer.

Due to its symmetry and nuclear spin statistics, only half of the normally expected rotational levels are allowed for the normal isotopologue, $\left({ }^{12} \mathrm{C}^{16} \mathrm{O}\right)_{2}$. It was experimentally established that the rotational levels occur in "stacks" and that every level within a given stack has the same symmetry: either $A^{+}$ or $A^{-}$. The presently known energy level stacks for the ground vibrational state of the $\mathrm{CO}$ monomers have been arbitrarily labeled $a, b, c, \ldots, k$, as shown in Figure 1. The stacks are characterized by well-defined values of $K(=0,1)$, the quantum number for the projection of the total angular momentum $J$ on the intermolecular axis. Pure rotational transitions within a stack are forbidden, and only transitions between $A^{+}$and $A^{-}$stacks are allowed.

A remarkable feature of the energy level scheme is the presence of two isomers in the form of two low-lying $A^{+}$stacks 


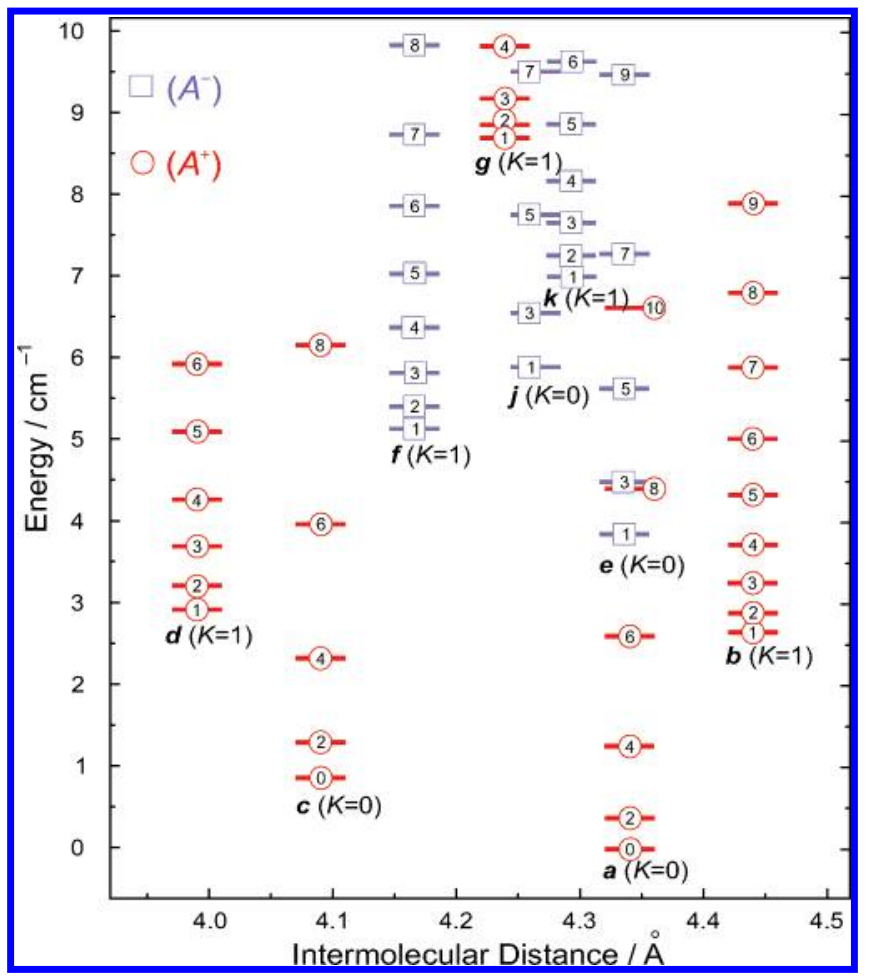

Figure 1. Ground state $\left(v_{\mathrm{CO}}=0\right)$ energy levels of the ${ }^{12} \mathrm{C}^{16} \mathrm{O}$ dimer determined in a previous study. ${ }^{17}$ The stacks of rotational levels are arbitrarily labeled $a, b, c$, etc. The levels are enclosed in circles for the $A^{+}$states and in squares for $A^{-}$states.

with $K=0$ (the ground $a$ state and the excited $c$ state at 0.88 $\mathrm{cm}^{-1}$ ). By comparison with theoretical calculations ${ }^{25,26}$ it was found that both isomers are planar with slipped antiparallel structures as shown in Figure 2. The $a$ state corresponds to the global minimum in the potential energy surface with the $\mathrm{C}$ atoms closer to each other than the $\mathrm{O}$ atoms. The $c$ state corresponds to a local minimum in the intermolecular potential where the $\mathrm{O}$ atoms are closer together. The effective intermolecular distance is larger for the C-bonded isomer (4.34 $\AA$ ) than for the O-bonded isomer ( $4.10 \AA$ ). In addition to these $K=0$ states, two $K=1$ stacks (designated as $b$ and $d$ ) have been assigned in the original IR studies of $\left({ }^{12} \mathrm{C}^{16} \mathrm{O}\right)_{2} \cdot{ }^{12,13}$

There is also $A^{+} / A^{-}$symmetry splitting (formerly believed to be a tunneling splitting), ${ }^{5,19}$ represented by the separation between the $A^{+}$ground state (the $a$ state) and the lowest $A^{-}$ stack (the $e$ state at $3.73 \mathrm{~cm}^{-1}$ with $K=0$ ). The ab initio studies ${ }^{25,26}$ have shown that this $A^{+} / A^{-}$separation corresponds to an antisymmetric vibration or geared bending motion of the complex. The very low excitation frequency indicates that the dimer is indeed very floppy. The discovery of the $A^{-}$levels was made by observations in the millimeter-wave region ${ }^{14}$ which were assigned with the help of infrared combination differences. All four stacks of $A^{-}$symmetry paired to the known stacks of $A^{+}$symmetry have been discovered, ${ }^{14-18}$ i.e. $e$ and $j$ states with $K=0$ and $f$ and $k$ states with $K=1$. Rovibrational calculations using a hybrid CCSD(T)/DFT-SAPT potential have reproduced all the observed stacks shown in Figure 1 in very good agreement with experiment. ${ }^{26}$

One of the interesting results of the previous millimeter-wave study ${ }^{17}$ was the observation of a higher energy stack of levels of $A^{+}$symmetry with $K=1$, denoted in Figure 1 as the $g$ stack. The origin of this stack at $8.50 \mathrm{~cm}^{-1}$ corresponds better to energy states with $K=2$ than to $K=1$. Recently, a similar situation was observed for the $K=1$ state in the $\mathrm{CO}-$ para $_{2}$ complex, ${ }^{27}$ which is isoelectronic to the $\mathrm{CO}$ dimer and has comparable binding energy. It was suggested that the monomers rotate nearly freely in the complex, with rotational quantum

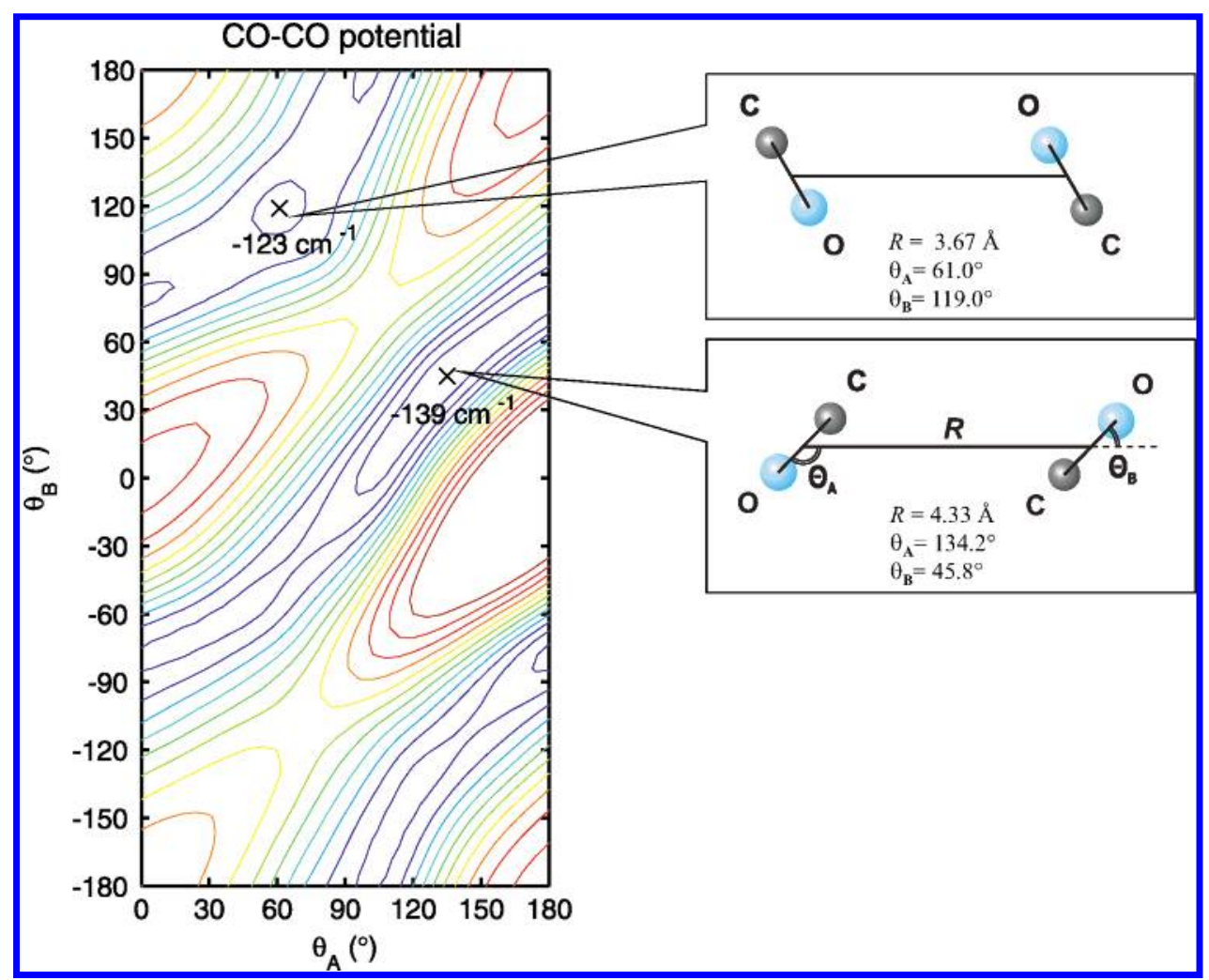

Figure 2. Cut through the hybrid CCSD(T)/DFT-SAPT potential ${ }^{26}$ (left panel). The coordinates given are for dihedral angle $\varphi=180^{\circ}$, with an extended range of $\theta_{B}$ so that all planar geometries are visible simultaneously. Negative $\theta_{B}$ is equivalent to taking $\varphi=0^{\circ}$. At each $\left(\theta_{A}, \theta_{B}\right)$ point the potential is minimized in the $R$ coordinate. The equilibrium structures of the CO dimer shown in the right panel correspond to the global minimum and the local minimum in the potential surface. 


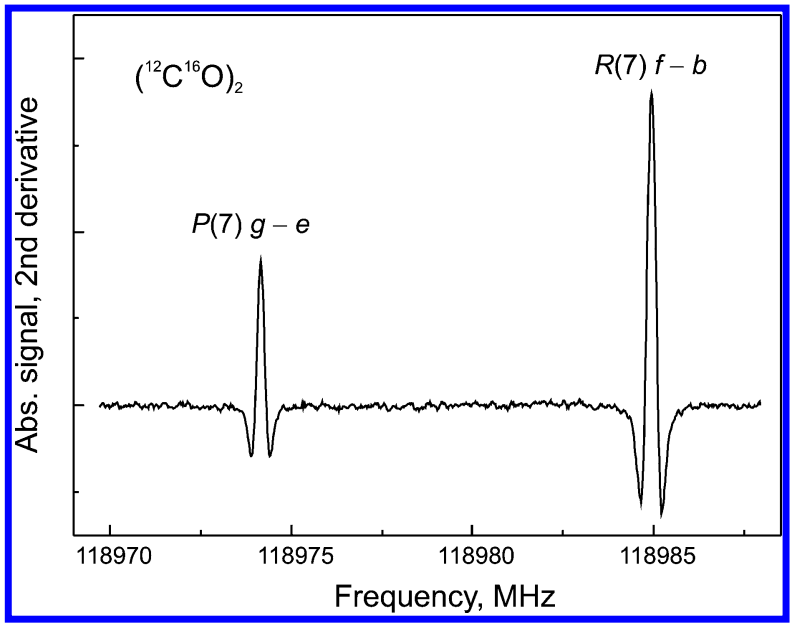

Figure 3. Two observed transitions of the $\mathrm{CO}$ dimer. The second derivative of the line shape is due to frequency modulation and second harmonics detection.

numbers $j_{1}=j_{2}=1$, and that the stack origins are defined by the energies of these rotations. In such a case we can expect even more stacks with different $K(=0,1,2)$ values located close to each other, as it was observed in the IR study of COpara $_{2}$ with $K=1$ and $2\left(v_{\mathrm{CO}}=1\right){ }^{28}$

To understand the nature of the $g$ state as well as to discover other high energy states of the $\mathrm{CO}$ dimer, we made a new observation of $\left({ }^{12} \mathrm{C}^{16} \mathrm{O}\right)_{2}$ spectra in the frequency range of $81-$ $135 \mathrm{GHz}$. In this study we used a "warm" molecular jet because the allowed transitions to the expected stacks originate from the levels with $A^{-}$symmetry which are not sufficiently populated in the cold jet conditions. The preliminary results of this observation were qualitatively considered already ${ }^{19}$ but at that time with an incorrect understanding of the $A^{+} / A^{-}$ splitting.

\section{Experimental Details}

The new millimeter-wave spectra of the ${ }^{12} \mathrm{C}^{16} \mathrm{O}$ dimer were measured in the frequency range from 81 to $135 \mathrm{GHz}$ using an intracavity jet spectrometer, OROTRON. Details of the setup are given elsewhere. ${ }^{29}$ Briefly, the homemade millimeter-wave generator OROTRON is placed in the vacuum chamber together with the supersonic jet apparatus. The molecular beam is injected into the OROTRON cavity perpendicularly to its axis. The high $Q$-factor of the cavity results in 100 effective passes of the radiation through the jet. Absorption in the cavity changes the generation conditions in the OROTRON and correspondingly the losses of the kinetic energy of electrons that can be detected very sensitively by measuring electron current changes in the collector circuit. A small part of the millimeter-wave radiation is taken out of the cavity through the coupling openings in a spherical mirror and mixed with the radiation of an HP 8671B microwave synthesizer for frequency determinations.

The experiment was performed with a pinhole pulsed jet source, General Valve, Series 9. The diameter of the nozzle was $0.8 \mathrm{~mm}$, and the typical repetition rate was $10-15 \mathrm{~Hz}$. The pumping system utilized an Edwards 9B3K Booster pump and as backing a Pfeiffer DUO 20M rotary pump. We used a gas mixture of $50 \%$ of $\mathrm{CO}$ and $50 \%$ of $\mathrm{Ne}$ or a pure $\mathrm{CO}$ gas at a backing pressure of 3.5 bar. Such a "warm" gas mixture resulted in dense and complicated but rather comprehensive spectra. For most of the absorption measurements, the full line width at halfheight (fwhh) is about $300 \mathrm{kHz}$ and the accuracy of the line center positions is estimated to be about $50 \mathrm{kHz}$.

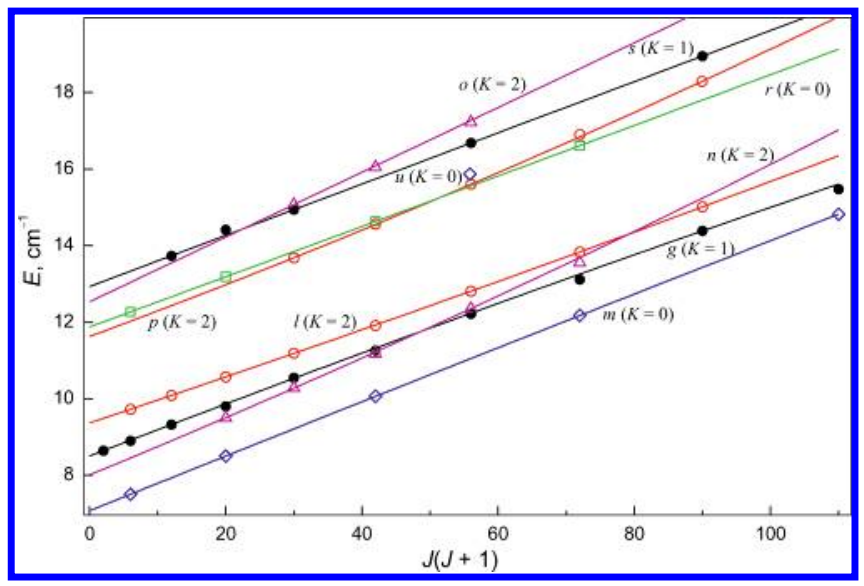

Figure 4. Dependence of the energy levels on total angular momentum for the newly observed stacks.

\section{Observations and Analysis}

More than 300 new spectral transitions including 27 transitions predicted by previous studies ${ }^{17}$ were measured in the frequency range $81-135 \mathrm{GHz}$. Our analysis involved these new transitions, the unassigned lines from our earlier search ${ }^{14,17}$ in the frequency range of $131-174 \mathrm{GHz}$, Klemperer's microwave data, ${ }^{1}$ and several lines in the range of $60-80 \mathrm{GHz}$ measured and put at our disposal by McKellar. ${ }^{30}$ The total number of transitions in the analysis was more than 600 . We used a simple "blind" combination difference approach. The computational program selected the pairs of unassigned transitions with differences or sums of their frequencies close to energy differences of any two precisely known energy levels that can be connected by electric dipole transitions to some new energy level. With the inclusion of these new levels in the existing energy level scheme the new transitions could be predicted and checked. As a rule, an energy level was considered as "confirmed" if at least three transitions were found which involve this level and provide an uncertainty in its position less than $100 \mathrm{kHz}$. Then the combination difference search among the unassigned lines continued involving the determined and the "confirmed" new energy level.

As a result 236 new transitions were assigned (360 transitions in total) and 53 new and 3 earlier predicted ${ }^{16,17}$ energy levels were precisely located (103 in total). The observed transitions and their assignments can be found in the Supporting Information. An example of a line recording is given in Figure 3. It demonstrates two lines of two different bands, i.e., $g-e, P(7)$, and $f-b, R(7)$, in one continuous scan from 118970 to 118987.5 MHz.

The energy levels are presented in Tables 1 and 2 with the new values given in bold face. Some of them extend previously observed stacks, but others compose the new higher energy stacks which are the subject of this paper.

(a) Higher- $J$ Transitions and Levels of $a, b, c, d$ States of $A^{+}$and $e, f, j$, and $k$ States of $A^{-}$Symmetry. Among the newly assigned levels 18 levels belong to already known stacks with higher $J$-values. In the previous study ${ }^{17}$ the maximum $J=8$ for the $a$ state was known unambiguously. Two other unverified $J=8$ energies were also given in earlier work, ${ }^{16}$ those for the $b$ and $c$ states. For these levels the situation changed: we were able to observe many transitions to confirm the tentative value of $b(8)$ and to change the unconfirmed value of $c(8)$ for a new one. We could also confirm tentative values ${ }^{17}$ for the $J=9$ level of the $e$ state and the $J=10$ level of the $a$ state. Other rotational energy levels determined for the first time with this 
TABLE 1: Experimental and Calculated (in Parentheses) Ground-State $\left(v_{\mathrm{CO}}=0\right) \boldsymbol{A}^{+}$Symmetry Energy Levels of the CO Dimer (in $\left.\mathrm{cm}^{-1}\right)^{a}$

\begin{tabular}{|c|c|c|c|c|c|c|c|c|c|}
\hline$J$ & $a(K=0)$ & $c(K=0)$ & $b(K=1)$ & $d(K=1)$ & $g(K=1)$ & $l(K=2)$ & $m(K=0)$ & $n(K=2)$ & $r(K=0)$ \\
\hline 0 & $0.000000(0.00)$ & $0.877208(0.89)$ & & & & & $\mathbf{7 . 0 7 6} 777$ (6.87) & & $\mathbf{1 1 . 8 7 4 2 5 8}(11.86)$ \\
\hline 1 & & & $2.654272(2.63)$ & $2.934585(2.83)$ & $8.644731(8.41)$ & & & & \\
\hline 2 & $0.382363(0.38)$ & $1.311855(1.31)$ & 2.893319 (2.87) & $3.227827(3.11)$ & $8.896271(8.66)$ & $9.724967 \quad(9.66)$ & $7.505377(7.29)$ & $(8.15)$ & $12.267404(12.25)$ \\
\hline 3 & & & $3.261678(3.25)$ & $3.710703(3.59)$ & $9.327586(9.09)$ & $\mathbf{1 0 . 0 8 7 9 1 5}(10.02)$ & & (8.62) & \\
\hline 4 & $1.258815(1.26)$ & $2.340127(2.33)$ & $3.725279(3.71)$ & $4.282513(4.15)$ & $9.802068(9.58)$ & $\mathbf{1 0 . 5 7 0} 770(10.50)$ & $8.502604(8.27)$ & $9.496205(9.24)$ & $\mathbf{1 3 . 1 8 5 0 5 5}(13.15)$ \\
\hline 5 & & & $4.344637(4.33)$ & $5.115318(4.97)$ & $10.547648(10.32)$ & $\mathbf{1 1 . 1 8 4 8 4 2}$ (11.11) & & $\mathbf{1 0 . 2 7 6 2 0 8}(10.01)$ & \\
\hline 6 & $2.607307(2.61)$ & 3.978964 (3.94) & $5.024614(5.01)$ & $5.945290(5.79)$ & $11.258067(11.05)$ & $11.908072(11.82)$ & $\mathbf{1 0 . 0 6 1 6 5 1}(9.81)$ & $11.177391(10.90)$ & $\mathbf{1 4 . 6 3 1} 449(14.58)$ \\
\hline 7 & & & 5.902305 & 7.144438 & 12.217865 & 12.804812 & & 12.354772 & \\
\hline 8 & 4.416749 & $6.222441^{b}$ & $6.803786^{c}$ & & 13.110130 & 13.757692 & 12.171572 & 13.552598 & 16.609347 \\
\hline 9 & & & 7.933985 & & 14.385634 & 15.009769 & & & \\
\hline 10 & $6.685416^{d}$ & & & & 15.473907 & & 14.816250 & & \\
\hline
\end{tabular}

${ }^{a}$ Values given in bold are new, relative to ref $17 .{ }^{b}$ Value is changed as compared to ref $15 .{ }^{c}$ Value from ref 16 , confirmed in this study. ${ }^{d}$ Value from ref 17 , confirmed in this study.

TABLE 2: Experimental and Calculated (in Parentheses) Ground-State $\left(v_{\mathrm{CO}}=0\right) \boldsymbol{A}^{-}$Symmetry Energy Levels of the CO Dimer (in $\left.\mathrm{cm}^{-1}\right)^{a}$

\begin{tabular}{|c|c|c|c|c|c|c|c|c|}
\hline $\mathrm{J}$ & $e(K=0)$ & $f(K=1)$ & $j(K=0)$ & $k(K=1)$ & $s(K=1)$ & $p(K=2)$ & $u(K=0)$ & $o(K=2)$ \\
\hline 1 & $3.859124(3.67)$ & $5.150544(5.26)$ & $5.905449(5.88)$ & 7.012535 (7.12) & (13.19) & & (12.94) & \\
\hline 2 & & $5.428450(5.54)$ & & 7.270475 (7.38) & (13.49) & (11.95) & & (12.19) \\
\hline 3 & $4.497930(4.32)$ & $5.836250(5.93)$ & $6.569876(6.53)$ & 7.674936 (7.78) & 13.733846 (13.89) & (12.34) & (13.59) & $(12.60)$ \\
\hline 4 & & $6.392050(6.49)$ & & $8.187246(8.29)$ & $\mathbf{1 4 . 4 1 0 8 9 3}(14.53)$ & (12.84) & & (13.16) \\
\hline 5 & $5.644308(5.48)$ & $7.047091(7.12)$ & $7.773892(7.71)$ & 8.879925 (8.96) & 14.938810 (15.14) & $\mathbf{1 3 . 6 8 1 0 7 4}(13.46)$ & (14.78) & $\mathbf{1 5 . 0 6 9} 370(13.88)$ \\
\hline 6 & & 7.883347 (7.96) & & $9.648682(9.73)$ & (16.18) & $\mathbf{1 4 . 5 6 2 5 2 1}(14.18)$ & & $\mathbf{1 6 . 0 5 0} 232$ (14.74) \\
\hline 7 & 7.289520 & 8.753408 & 9.523951 & 10.649019 & 16.677285 & 15.596154 & 15.851151 & 17.267221 \\
\hline 8 & & 9.871211 & & 11.681819 & & 16.899715 & & \\
\hline 9 & $9.418969^{b}$ & 10.930577 & 11.820991 & & 18.949506 & 18.288498 & & \\
\hline
\end{tabular}

${ }^{a}$ Values given in bold are new, relative to ref $17 .{ }^{b}$ Value from ref 17 , confirmed in this study. 
TABLE 3: Observed Millimeter-Wave Transitions on Two Selected Energy Levels, $f(4)$ and $f(5)$

\begin{tabular}{|c|c|c|c|c|c|}
\hline \multicolumn{3}{|c|}{$f(4)$} & \multicolumn{3}{|c|}{$f(5)$} \\
\hline energy level & freq, $\mathrm{MHz}$ & diff, $\mathrm{MHz}^{a}$ & energy level & freq, $\mathrm{MHz}$ & $\overline{\operatorname{diff}, \mathrm{MHz}^{a}}$ \\
\hline$a(4)$ & 153890.572 & 0.048 & $a(4)$ & 173528.088 & -0.038 \\
\hline$c(4)$ & 121473.630 & 0.010 & $a(6)$ & 133101.355 & -0.011 \\
\hline$b(3)$ & 93846.183 & -0.008 & $c(4)$ & 141111.267 & 0.044 \\
\hline$b(4)$ & 79947.788 & -0.006 & $c(6)$ & 91980.134 & -0.020 \\
\hline$b(5)$ & 61379.902 & -0.035 & $b(4)$ & 99585.388 & -0.009 \\
\hline$d(3)$ & 80384.773 & 0.029 & $b(5)$ & 81017.519 & -0.021 \\
\hline$d(5)$ & 38275.519 & 0.054 & $d(6)$ & 33031.145 & -0.026 \\
\hline$g(3)$ & 88005.139 & 0.012 & $g(4)$ & 82592.093 & -0.041 \\
\hline$g(4)$ & 102229.770 & 0.032 & $g(5)$ & 104944.078 & 0.007 \\
\hline$g(5)$ & 124581.672 & -0.001 & $g(6)$ & 126241.870 & -0.059 \\
\hline$l(3)$ & 110799.249 & 0.033 & $l(4)$ & 105637.243 & -0.051 \\
\hline$l(4)$ & 125274.856 & -0.041 & $l(5)$ & 124046.692 & 0.043 \\
\hline$l(5)$ & 143684.300 & 0.048 & $m(6)$ & 90374.256 & 0.025 \\
\hline$n(4)$ & 93060.235 & 0.019 & $n(5)$ & 96806.519 & 0.015 \\
\hline$n(5)$ & 116444.095 & -0.011 & $n(6)$ & 123823.290 & -0.006 \\
\hline
\end{tabular}

${ }^{a}$ The difference between the observed frequency and the frequency calculated from the energy levels as determined in Tables 1 and 2.

TABLE 4: Empirical and Calculated (in Parentheses) Parameters for the Observed States of the CO Dimer

\begin{tabular}{|c|c|c|c|c|c|c|c|}
\hline \multirow{2}{*}{$\begin{array}{c}\text { state } \\
a\end{array}$} & \multicolumn{2}{|c|}{ origin, $\mathrm{cm}^{-} 1$} & $B, \mathrm{~cm}^{-1}$ & \multicolumn{2}{|c|}{$D, \mathrm{~cm}^{-1}$} & \multirow{2}{*}{$\begin{array}{l}H, \mathrm{~cm}^{-1} \\
1.7 \times 10^{-7}\end{array}$} & \multirow{2}{*}{$\frac{R_{\text {eff }}, \AA}{4.35(4.34)}$} \\
\hline & 0.0 & & $0.0637 \quad(0.0638)$ & $4.6 \times 10^{-5}$ & $\left(4.1 \times 10^{-5}\right)$ & & \\
\hline$b$ & 2.528 & $(2.504)$ & $0.0614 \quad(0.0622)$ & $5.7 \times 10^{-5}$ & $\left(5.7 \times 10^{-5}\right)$ & $4.5 \times 10^{-7}$ & $4.43(4.40)$ \\
\hline$c$ & 0.8771 & $(0.886)$ & $0.0722(0.0714)$ & $-5.6 \times 10^{-5}$ & $\left(-3.4 \times 10^{-5}\right)$ & $-3.7 \times 10^{-7}$ & $4.09(4.11)$ \\
\hline$d$ & 2.792 & (2.658) & $0.0740 \quad(0.0775)$ & $-5.9 \times 10^{-5}$ & $\left(6.1 \times 10^{-5}\right)$ & & $4.03(3.94)$ \\
\hline$e$ & 3.7306 & $(3.542)$ & $0.0641 \quad(0.0647)$ & $1.0 \times 10^{-5}$ & $\left(1.1 \times 10^{-5}\right)$ & & $4.34(4.31)$ \\
\hline$f$ & 5.029 & (5.134) & $0.0662 \quad(0.0665)$ & $-7.0 \times 10^{-5}$ & $\left(-1.2 \times 10^{-5}\right)$ & $-8.5 \times 10^{-7}$ & $4.27(4.26)$ \\
\hline$j$ & 5.7728 & $(5.747)$ & $0.0662(0.0648)$ & $-1.8 \times 10^{-5}$ & $\left(-1.8 \times 10^{-5}\right)$ & $-7.9 \times 10^{-7}$ & $4.26(4.31)$ \\
\hline$k$ & 6.874 & (6.990) & $0.0662 \quad(0.0653)$ & $-1.0 \times 10^{-5}$ & $\left(-3 \times 10^{-6}\right)$ & & $4.27(4.30)$ \\
\hline$m$ & 7.07672 & $2(6.866)$ & $0.07152(0.0705)$ & $1.1 \times 10^{-5}$ & $\left(8.6 \times 10^{-6}\right)$ & & $4.10(4.13)$ \\
\hline$n$ & 7.920 & (7.678) & $0.07860(0.0790)$ & $2.0 \times 10^{-6}$ & $\left(5.3 \times 10^{-5}\right)$ & & $3.91(3.90)$ \\
\hline$g$ & 8.511 & $(8.258)$ & $0.0664 \quad(0.0692)$ & $3.9 \times 10^{-5}$ & $\left(6.0 \times 10^{-5}\right)$ & & $4.21(4.17)$ \\
\hline l & 9.385 & $(9.301)$ & $0.0582 \quad(0.0600)$ & $-4.5 \times 10^{-5}$ & $\left(-1.7 \times 10^{-6}\right)$ & & $4.55(4.48)$ \\
\hline$o$ & 12.869 & (11.773) & $0.06740(0.0686)$ & $-2.0 \times 10^{-4}$ & $\left(-5.1 \times 10^{-5}\right)$ & & $4.23(4.19)$ \\
\hline$s$ & 13.00 & (13.072) & $0.0655(0.0666)$ & $-6.0 \times 10^{-6}$ & $\left(-1.6 \times 10^{-4}\right)$ & & $4.29(4.25)$ \\
\hline$p$ & 11.53 & (11.567) & $0.0697 \quad(0.0650)$ & $-6.1 \times 10^{-5}$ & $\left(6.7 \times 10^{-5}\right)$ & & $4.16(4.30)$ \\
\hline$u$ & & (12.804) & $(0.0657)$ & & $\left(-8.8 \times 10^{-6}\right)$ & & $(4.28)$ \\
\hline$r$ & 11.874 & (11.857) & $0.0655(0.0648)$ & $-4.1 \times 10^{-6}$ & $\left(-2.5 \times 10^{-6}\right)$ & & $4.29(4.31)$ \\
\hline
\end{tabular}

study are $b(9), d(7), f(8,9,10), j(9)$, and $k(7,8)$. The frequency of the $b(7)-f(7)$ transition given in ref 17 was incorrect because of a misprint. The correct frequency is $85473.889 \mathrm{MHz}$.

All of these levels are linked by three or more transitions (for example, the $f(8)$ level is linked by 8 transitions), and their assignment is unambiguous.

(b) $g, l, m, n$, and $r$ Stacks: New Higher Energy $A^{+}$States. The $g$ state with $K=1$ is not really a new stack. It was discovered in our previous work, ${ }^{17}$ but for reasons of consistency we will consider this stack together with the newly observed states. The earlier observed $g$ state levels, with $J=1-4$ inclusive, were located above $8.5 \mathrm{~cm}^{-1}$, which is considerably higher in energy than any of the four previously characterized $A^{+}$states. In this study we have found higher $J$ levels up to 10 (see Table 1). The lower levels in transitions to the $g$ state belong to the $e, f$, and $j$ states but not to the $k$ state, since such lines would lie at lower frequencies $(27-64 \mathrm{GHz})$.

By the application of a combination difference analysis to the observed lines, we discovered for the first time a $K=2$ energy stack. It was located at $9.368 \mathrm{~cm}^{-1}$ and was labeled as $l$ stack. The $K$ value of 2 was chosen because we could not find any proper transition linking the potential $l(1)$ level, although the next $l(2)$ level was involved in 6 transitions. The highest energy level observed for the new $l$ stack has $J=9$. The most prominent subbands are $l-f, K=2-1$ and $l-j, K=$ $2-0$, including all $P-, Q-$, and $R$-branches. The $R$-branch of $l-k, K=2-1$, is also complete, but the $P$ - and $Q$-branches would lie at lower frequencies $(33-73 \mathrm{GHz})$. Three transitions connecting $l$ and $e$ states were also detected.

Further analysis of the combination differences of the observed lines resulted in the discovery of two $K=0$ stacks ( $m, J=0-10 ; r, J=0-8)$. The $m$ state is located at 7.077 $\mathrm{cm}^{-1}$, and the $r$ state at $11.874 \mathrm{~cm}^{-1}$. Most of the levels of these stacks are connected with other levels by three or more transitions. Exceptions are the $r(2)$ and $r(8)$ levels which are connected by two transitions and the $m(0)$ and $r(0)$ levels which are connected by only one transition. Nevertheless, these levels were accepted because (1) their positions are fitted very well by the standard $J(J+1)$ expression for the corresponding stack and (2) the intensities of transitions to these levels correlate very well with the intensities of other transitions within the same branches or subbands. A further experimental test would be useful, but all other possible transitions to these levels are out of the frequency range of our spectrometer.

One more stack with $K>0$ was derived from the analysis of the observed transitions. For this stack we were not able to detect the lowest energy level, but from its location and the existence of odd- $J$ levels the expected $K$ value was 1 or 2 . As we show below the more probable value is $K=2$. This new stack was labeled by $n$ and involved energy levels with $J=$ $4-8$. The estimated frequencies of some low- $J$ transitions are in the range of our spectrometer, but we were unable to detect 

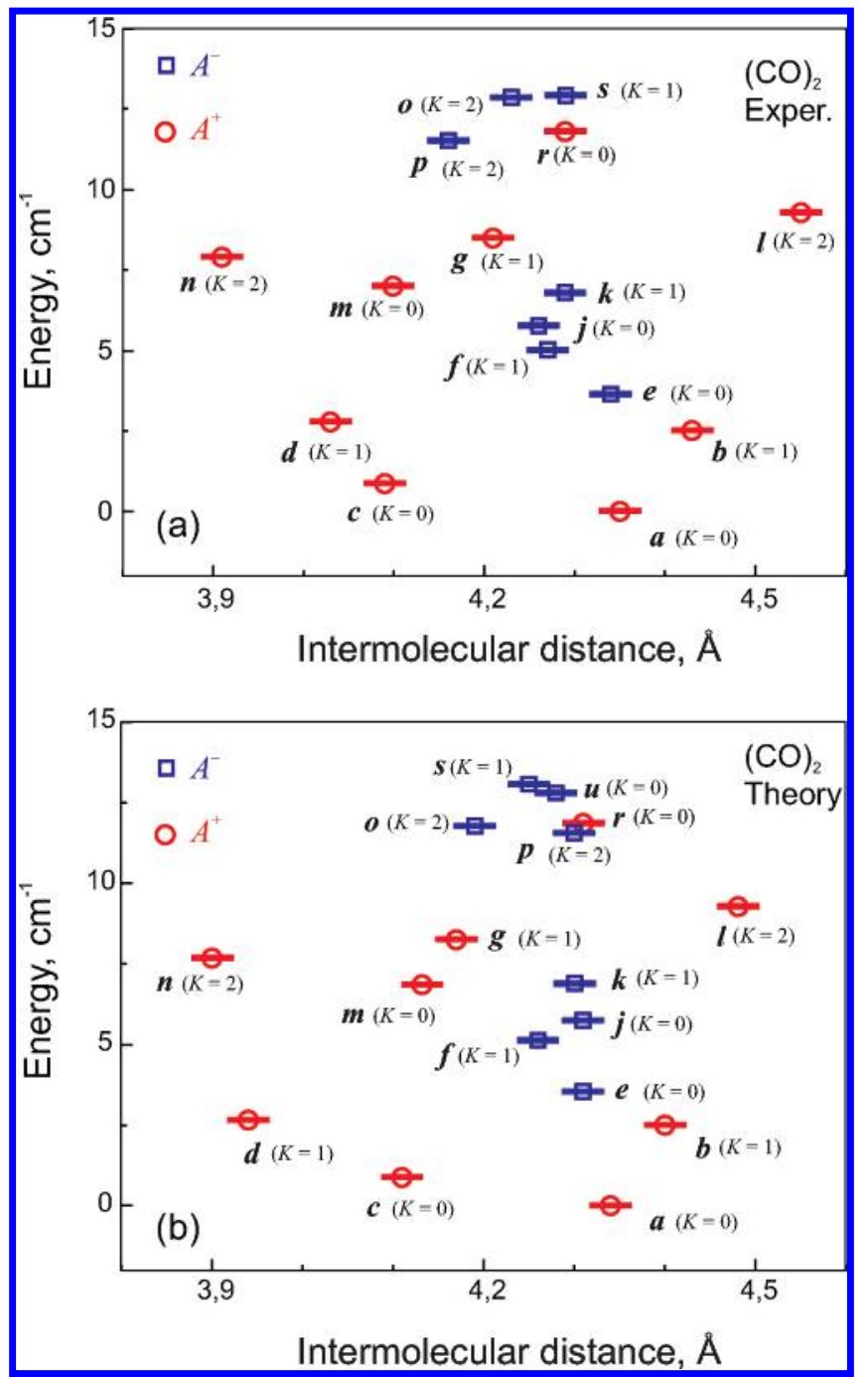

Figure 5. Stack origins of the $\mathrm{CO}$ dimer plotted against the intermolecular separation as determined from rotational spacing: (a) experiment; (b) theory. The stack origins are enclosed in circles for the $A^{+}$states and in squares for $A^{-}$states. The $u$-stack is not presented on the experimental plot because only one $J=7$ level has been detected.

them even when searching carefully. Higher- $J$ transitions connecting the $n$ stack with lower $e, j$, and $f$ stacks were weakly observed as well as two transitions connecting $n(8)$ level with $o(7)$ and $p(9)$ levels of higher $A^{-}$states discussed in the next section. Hence, the $n$ stack is the only one that is incomplete among all observed stacks with $A^{+}$symmetry.

(c) $o, p, s$, and $u$ Stacks: New Higher Energy $A^{-}$States. A total of 14 new levels of $A^{-}$symmetry were found that are relatively high: from 13.73 to $18.95 \mathrm{~cm}^{-1}$. The transitions to these levels are "hot", and their intensity in the jet is rather low. However, to every reported level three or more linking transitions were detected, with the exception of the $p(9)$ level linked by two transitions.

The attribution of these 14 levels to certain stacks was not straightforward because we could not detect the levels with small $J$ values. Only one level with $J=3$ and one with $J=4$ (finally assigned as $s(3)$ and $s(4))$ were found. Some higher- $J$ levels were also missing. Nevertheless we were able to arrange these levels into four stacks. Three of them should have $K>0$, because of the existence of levels with even $J$ values. The $K$ values for these new stacks of $A^{-}$symmetry have been assigned by establishing relations between them and the stacks with $A^{+}$ symmetry.
It was quite helpful to look at the dependence of the energy of new higher located states on $J(J+1)$ as shown in Figure 4. One can see that the levels can be easily separated into different stacks for which this dependence is close to linear. In such a way we plotted five new states of $A^{+}$symmetry: two with $K$ $=0(\mathrm{~m}$ and $r)$; one with $K=1(\mathrm{~g})$; one with $K=2(l)$; one with $K=1$ or $2(n)$. And one observes three states of $A^{-}$ symmetry with $K>0$. A specific feature visible in Figure 4 is the crossing of two stacks: $g$ and $n$ at $J=6-7$. A similar crossing occurs for the $o$ and $s$ states of $A^{-}$symmetry at $J=$ $4-5$. It is natural to suppose a correlation between these two crossings and to give corresponding $K$ values to the stacks of $A^{-}$symmetry. Both the $o$ and $s$ stacks, but in different numbers, involve transitions from $g$ and $l, m, n$ stacks. The remaining stack of $A^{-}$symmetry in Figure 4 denoted as $p$ was attributed to $K=$ 2. It involves different and incomplete branches of transitions originating from the four newly observed stacks of $A^{+}$symmetry.

There is also one separate level located at energy of 15.851 $\mathrm{cm}^{-1}$. Its $J$-value is ambiguous (7 or 8 ) because we could observe only 3 transitions from $g(7), l(7)$, and $n(7)$ and 2 transitions from $g(8)$ and $m(8)$ levels. Nevertheless, simply assuming a parity between $A^{+}$and $A^{-}$states with similar $K$-values it was assigned as $J=7$ level and attributed to the missing $u$ stack with $K=0$.

(d) Energy Levels. All of the unambiguously assigned observed transitions of $\left({ }^{12} \mathrm{C}^{16} \mathrm{O}\right)_{2}$ listed in the Supporting Information were combined to determine rotational energy levels for the dimer with the aid of a least-squares term value program. All established energy levels are presented in Tables 1 and 2. Due to the high precision of the millimeter-wave line positions, the nature of the transitions, and the energy level structure, these values are unambiguous, model-independent, and very precise $\left(\approx 0.1 \mathrm{MHz}\right.$, or $\left.0.000003 \mathrm{~cm}^{-1}\right)$.

As an example of the consistency of our assignment and verification procedure, we present the observed linking transitions for two selected energy levels, i.e., $f(4)$ and $f(5)$ in Table 3 . The first column shows all energy states for which the connecting transitions with the $f(4)$ and $f(5)$ levels were found, the second one gives the measured frequencies of the transitions, and the third column illustrates the deviation of the observed frequencies from the values calculated from the energy levels in Tables 1 and 2 .

The levels of each stack were fitted with a simple semirigid rotor energy level expression

$$
\text { energy }=\text { origin }+B J(J+1)-D[(J(J+1))]^{2}
$$

For some stacks of levels, the earlier analysis also included the effect of a Coriolis-type interaction term, $W_{\mathrm{C}} J(J+1)$, linking pairs of states. But due to the very close location and more complicated mixing of the newly observed states, we did not attempt to account for such an interaction here. By contrast, we included a higher centrifugal term $H\left[(J(J+1)]^{3}\right.$ fitting some stacks with high- $J$ levels if the number of data was sufficient and if such an addition improved essentially the fit quality. The results are listed in Table 4. From the values of the rotational constant $B$ intermolecular distances $R_{\text {eff }}$ values between the monomers were determined for each state of the dimer.

\section{Rovibrational Calculations}

As in refs 25 and 26 we define the geometry of the $\mathrm{CO}$ dimer consisting of molecules $A$ and $B$ by the four coordinates $R, \theta_{A}$, $\theta_{B}$, and $\varphi$. The intermolecular distance $R$ is the length of the 


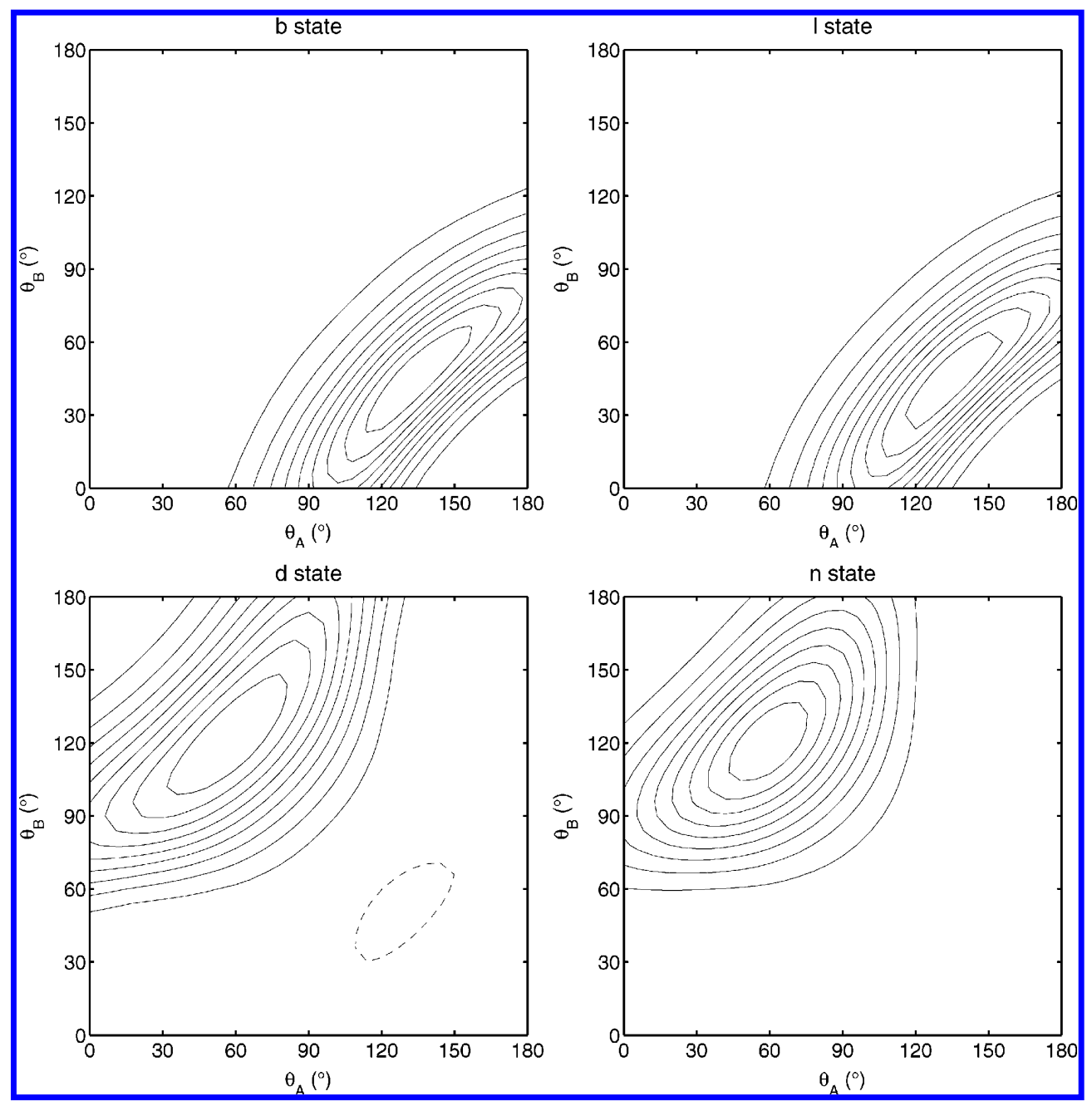

Figure 6. Internal wave functions of the previously detected $b$ and $d$ states with $K=1$ and the new $l$ and $n$ states with $K=2$. The dihedral angle $\varphi$ was chosen to be $180^{\circ}$, and $R$ was fixed at the $R_{\text {eff }}$ value of each state. The wave functions $\psi$ for $K \neq 0$ depend on the overall rotation angles of the dimer; these were chosen such that the wave functions have substantial amplitudes. Contours are drawn at values $\pm k|\psi|_{\max } / 10$ for $k=$ $1, \ldots, 9$.

vector from the center of mass of monomer $A$ to that of monomer $B, \theta_{A}$, and $\theta_{B}$ are the angles between $\mathbf{R}$ and the vectors $\mathbf{r}_{A}$ and $\mathbf{r}_{B}$ pointing from the $\mathrm{C}$-atom to the $\mathrm{O}$-atom in the monomers, and $\varphi$ is the dihedral angle between the planes defined by $\mathbf{R}, \mathbf{r}_{A}$, and $\mathbf{r}_{B}$. The potential energy surface used in the calculations was the hybrid potential constructed in ref 26 . This potential shows two minima close in energy, both corresponding to planar slipped antiparallel structures. The global minimum at $-139 \mathrm{~cm}^{-1}$ corresponds to a structure where the $\mathrm{C}$ atoms are closer together, and the second minimum is 16 $\mathrm{cm}^{-1}$ higher in energy to a similar structure with the $\mathrm{O}$ atoms nearest to each other; see Figure 2. Due to zero-point energy effects, the rovibrational energies of the lowest two states, $a$ and $c$, that are more or less localized at these two minima are very close: the calculated energy difference is only $0.89 \mathrm{~cm}^{-1}$ for the ${ }^{12} \mathrm{C}^{16} \mathrm{O}$ dimer. The situation is complicated because this energy difference is so small that the $a$ and $c$ states are not completely localized at their respective minima but tunnel to some extent to the other minimum. Isotope substitutions, replacing ${ }^{12} \mathrm{C}$ by ${ }^{13} \mathrm{C}$ or ${ }^{16} \mathrm{O}$ by ${ }^{18} \mathrm{O}$, have a substantial effect on this tunneling. ${ }^{24,26}$

The method used for the rovibrational calculations is the same as in refs 25 and 26, and also the parameters and basis sets were the same. Rovibrational states of $A^{+}$and $A^{-}$symmetry were calculated for total angular momentum $J=0, \ldots, 6$, taking Coriolis coupling into account. Although $K$, the projection of the total angular momentum $J$ on $\mathbf{R}$, is strictly not a good quantum number, we found that for nearly all computed states the contribution of one particular $K$ value was dominant. The calculated energy levels are given in Tables 1 and 2 together with the experimentally deduced levels. As in the experiment, the calculated energy levels were fit to a simple rigid rotor expression. The resulting parameters are included in Table 4 with the experimentally deduced values. To facilitate comparison between theory and experiment, the stack origins are plotted against the effective intermolecular distances, $R_{\text {eff }}$, that were extracted from the rotational constants $B$ of each stack. Figure 5 a shows the measured data, and Figure $5 \mathrm{~b}$, the calculated values. The zero point of energy is chosen at the $a$ stack origin. It is obvious from Tables 1 and 2 and Figure 5a,b that theory and experiment agree very well also for the higher states, except for the origin of the $o$ stack and the value of $R_{\text {eff }}$ of the $p$ stack. The agreement is similarly good as it was for the lower states of $\left({ }^{12} \mathrm{C}^{16} \mathrm{O}\right)_{2}$ observed previously and for the isotopologues $\left({ }^{13} \mathrm{C}^{16} \mathrm{O}\right)_{2}$ and $\left({ }^{12} \mathrm{C}^{18} \mathrm{O}\right)_{2} \cdot{ }^{24,26}$ This shows that the hybrid potential constructed in ref 26 is very accurate indeed.

\section{Discussion}

The stack origins of the new $A^{+}$states $(m, n, g, l, r)$ range from 7 to $12 \mathrm{~cm}^{-1}$. The variation of the intermolecular separation for the different stacks (from 4.0 to $4.5 \AA$ ) is even larger than 


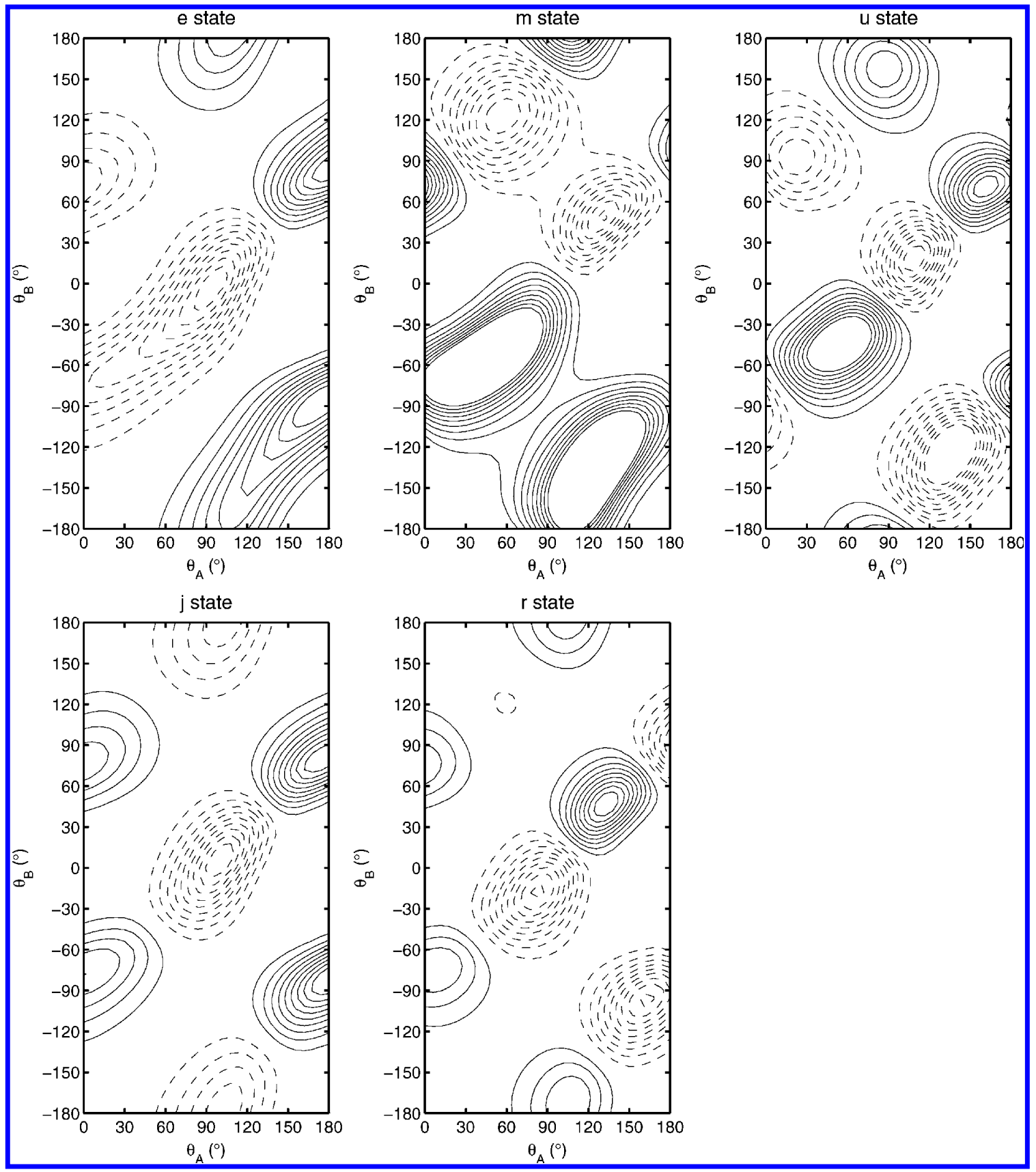

Figure 7. Internal wave functions of the $e$ and $j$ geared bend fundamental $\left(v_{\mathrm{b}}=1\right)$ states of $A^{-}$symmetry, the $m$ and $r$ overtone $\left(v_{\mathrm{b}}=2\right)$ states of $A^{+}$symmetry, and the $u$ second overtone $\left(v_{\mathrm{b}}=3\right)$ state of $A^{-}$symmetry. All these states have $K=0$. The dihedral angle $\varphi$ was chosen to be $180^{\circ}$ with an extended range of $\theta_{B}$ values that shows all planar geometries (cf. the potential in Figure 2), and $R$ was fixed at the $R_{\text {eff }}$ value of each state.

for the lower stacks, and the values of $R_{\text {eff }}$ are somewhat larger on average. The stack origins of the $A^{-}$states $(u, o, p, s)$ range from 11 to $13 \mathrm{~cm}^{-1}$. The intermolecular separation varies from 3.9 to $4.3 \AA$ for these stacks. Some of the new stacks have $K=$ 2, while the previously found stacks had $K=0$ and 1 only. The calculated values of $K$ confirm the experimental assignment in all cases. The wave functions of the previously detected $b$ and $d$ states with $K=1$ and the new $l$ and $n$ states with $K=2$ are plotted in Figure 6 . They are very similar to those of the $a$ and $c$ states in Figure 11 of ref 26. So, we interpret the $a, b$, and $l$ series and the $c, d$, and $n$ series as due to the rotation of the whole dimer about the long axis, with angular momenta $K$ $=0,1$, and 2 along this axis. The levels within each of the stacks are also due to rotation, of course, with increasing values of $J \geq K$. The corresponding dimer rotational constant $A$ is of approximately the same size as the $\mathrm{CO}$ monomer rotational constant, which can be understood if one looks at the dimer geometries shown in Figure 2. The parameter $A$ which could be derived from the splittings between the levels with different $K$ is not constant, however, because the complex is quite flexible.

In ref 26 it was explained that the $e$ and $j$ states of $A^{-}$ symmetry with $K=0$ correspond to a geared bend vibration. They are the fundamentals of this vibration, and their wave functions with a node at $\theta_{A}=180^{\circ}-\theta_{B}$ for $\varphi=180^{\circ}$ are shown (again) in Figure 7. From the wave functions of the new $m$ and $r$ states of $A^{+}$symmetry with $K=0$ also shown in Figure 7 , it follows that these states are geared bend overtones. So we have the two geared bend series: $e, m$ and $j, r$ with bend quantum number $v_{\mathrm{b}}=1$ and 2 , respectively, and $K=0$. The new $u$ state of $A^{-}$symmetry with $K=0$ is probably the second overtone with $v_{\mathrm{b}}=3$ in the first series; see Figure 7. The corresponding second overtone of the second series lies above 
TABLE 5: Rovibrational Assignment of the Observed Stacks of the CO Dimer $\left(v_{\mathrm{b}}=\right.$ Quantum Number Associated with the Geared Bend Mode; $K=$ Component of Total Angular Momentum along the Intermolecular Axis)

\begin{tabular}{ccccc}
\hline stack & origin, $\mathrm{cm}^{-1}$ & symmetry & $v_{\mathrm{b}}$ & $K$ \\
\hline$a$ & 0.0 & $A^{+}$ & 0 & 0 \\
$c$ & 0.88 & $A^{+}$ & 0 & 0 \\
$b$ & 2.53 & $A^{+}$ & 0 & 1 \\
$d$ & 2.79 & $A^{+}$ & 0 & 1 \\
$l$ & 9.39 & $A^{+}$ & 0 & 2 \\
$n$ & 7.92 & $A^{+}$ & 0 & 2 \\
$e$ & 3.73 & $A^{-}$ & 1 & 0 \\
$j$ & 5.77 & $A^{-}$ & 1 & 0 \\
$f$ & 5.03 & $A^{-}$ & 1 & 1 \\
$k$ & 6.87 & $A^{-}$ & 1 & 1 \\
$p$ & 11.53 & $A^{-}$ & 1 & 2 \\
$o$ & 12.87 & $A^{-}$ & 1 & 2 \\
$m$ & 7.08 & $A^{+}$ & 2 & 0 \\
$r$ & 11.87 & $A^{+}$ & 2 & 0 \\
$g$ & 8.51 & $A^{+}$ & 2 & 1 \\
$u$ & $12.80^{a}$ & $A^{-}$ & 3 & 0 \\
$s$ & 13.00 & $A^{-}$ & 3 & 1
\end{tabular}

${ }^{a}$ Calculated value.

the range of observation. The $R_{\text {eff }}$ values of these series are rather irregular. Figure 7 illustrates that this is because the geared bend excited states are no longer localized at one of the two minima, as the $a$ and $c$ ground states are. We noticed this already ${ }^{26}$ for the fundamentals $e$ and $j$. It can be understood because the "reaction path" between the two minima in the potential energy surface (with the lowest saddle point) follows the geared bend coordinate and has only a small energy barrier; see Figure 2.

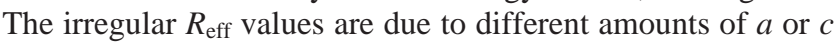
"character" of the bend excited states.

The internal wave functions of the $f$ and $k$ states of $A^{-}$ symmetry with $K=1$ and of the $p$ and $o$ states of $A^{-}$symmetry with $K=2$ are very similar to those of the $e$ and $j$ states, so these states also correspond to the geared bend fundamental, with $K(=1$ or 2$)$ quanta of the dimer overall rotation along the long axis. The calculated origin of the $o$ stack is somewhat lower than the experimental value. For the $p$ state with $K=2$ there is some disagreement between the measured and calculated $R_{\text {eff }}$ values; see Figure 5. The two minima in the potential have very different values of $R_{\mathrm{e}}$, and the $R_{\text {eff }}$ value of a state depends sensitively on its relative weight in either of the two minima. The two minima have zero point levels $a$ and $c$ with very nearly the same energy, so the relative $a$ and $c$ "character" of the higher states is very subtle. The internal wave function of the $g$ state of $A^{+}$symmetry with $K=1$ is similar to that of the $m$ state of $A^{+}$symmetry with $K=0$, so $g$ is a geared bend overtone, just like $m$, but with one quantum of dimer overall rotation along the long axis. The highest state of $A^{-}$symmetry, the $s$ state, is the $K=1$ counterpart of the second $\left(v_{\mathrm{b}}=3\right)$ bend overtone, the $u$ state with $K=0$. Its internal wave function is similar to that of the latter, shown in Figure 7. The assignment of all stacks observed is summarized in Table 5.

\section{Conclusions}

From the measurement and assignment of more than 300 new spectral transitions in the frequency range from 81 to $135 \mathrm{GHz}$, in combination with earlier data, we could deduce the precise location of 61 energy levels of $A^{+}$symmetry and 42 levels of $A^{-}$symmetry of the ${ }^{12} \mathrm{C}^{16} \mathrm{O}$ dimer. These energy levels, which correspond to intermolecular vibrations and rotations of this weakly bound van der Waals complex, can be arranged in 9 stacks of $A^{+}$symmetry and 8 stacks of $A^{-}$symmetry, with origins up to $13 \mathrm{~cm}^{-1}$ above the zero-point level. The energy levels from accompanying rovibrational calculations with the use of a recently developed hybrid CCSD(T)/DFT-SAPT potential are in very good agreement with experiment and confirm the assignments made. From the calculated wave functions, we deduce that the observed levels belong either to the ground $\left(A^{+}\right.$symmetry) state of two "isomers" located in different minima of the potential energy surface or to the geared bend mode of the $\mathrm{CO}$ dimer with overtones up to $v_{\mathrm{b}}=3$ excited. Geared bend states with odd $v_{\mathrm{b}}$ have $A^{-}$symmetry; those with even $v_{\mathrm{b}}$ have $A^{+}$symmetry. All of the geared bend excited states are strongly delocalized over the two "isomers". Different series of stacks correspond to overall rotations of the dimer with $K=$ 0,1 , and 2 , where $K$ is the angular momentum component along the intermolecular axis.

Acknowledgment. We are grateful to A. R. W. McKellar for providing the lower frequency millimeter-wave data before publication. L.A.S. and B.S.D. thank A. V. Potapov for his help in some additional verifying measurements. The work of D.N.F. and B.S.D. at Cologne was made possible by the DFG through a grant aimed to support Eastern and Central European Countries and Republics of the FSU (436RUS113/719/0-2) and of V.A.P. through the Leonhard-Euler Fellowship Program by the DAAD (A04/00926). The support of the Russian Foundation for Basic Research (RFBR: 06-02-16035) is gratefully acknowledged by L.A.S., B.S.D., and V.A.P.

Supporting Information Available: The observed millimeter-wave transitions of $\left({ }^{12} \mathrm{C}^{16} \mathrm{O}\right)_{2}$ and their assignments (Tables $1 \mathrm{~S}-12 \mathrm{~S}$ ), where in all tables the frequencies given with three digits were measured with an accuracy of $50 \mathrm{kHz}$, the frequencies given with two digits were measured with an accuracy of $100-150 \mathrm{kHz}$, and the predicted values are shown in parentheses. This material is available free of charge via the Internet at http://pubs.acs.org.

\section{References and Notes}

(1) Vanden Bout, P. A.; Steed, J. M.; Bernstein, L. S.; Klemperer, W. Astrophys. J. 1979, 234, 503

(2) Havenith, M.; Petri, M.; Lubina, C.; Hilpert, G.; Urban, W. J. Mol. Spectrosc. 1994, 167, 248

(3) Roth, D. A.; Hepp, M.; Pak, I.; Winnewisser, G. Chem. Phvs. Lett 1998, 298, 381.

(4) van der Pol, A.; van der Avoird, A.; Wormer, P. E. S. J. Chem. Phys. 1990, 92, 7498 .

(5) Bunker, P. R.; Jensen, P.; Althorpe, S. C.; Clary, D. C. J. Mol. Spectrosc. 1993, 157, 208.

(6) Meredith, A. W.; Stone, A. J. J. Phvs. Chem. 1998, 102, 434.

(7) Rode, M.; Sadlej, J.; Moszynski, R.; Wormer, P. E. S.; van der Avoird, A. Chem. Phys. Lett. 1999, 314, 326.

(8) Pedersen, T. B.; Fernández, B.; Koch, H. Chem. Phys. Lett. 2001 334,419 .

(9) Rode, M.; Sadlej, J.; Moszynski, R.; Wormer, P. E. S.; van der Avoird, A. Chem. Phys. Lett. 2001, 334, 424.

(10) Fišer, J.; Boublík, T; Polák, R. Mol. Phvs. 2003, 101, 3409.

(11) Burenin, A. V. Opt. Spectrosc. 2003, 95, 192.

(12) Brookes, M. D.; McKellar, A. R. W. Chem. Phys. Lett. 1998, 287, 365.

(13) Brookes, M. D.; McKellar, A. R. W. J. Chem. Phys. 1999, 111, 7321.

(14) Roth, D. A.; Surin, L. A.; Dumesh, B. S.; Winnewisser, G.; Pak, I. J. Chem. Phys. 2000, 113, 3034.

(15) Walker, K. A.; Xia, C.; McKellar, A. R. W. J. Chem. Phys. 2000 113,6618

(16) Walker, K. A.; McKellar, A. R. W. J. Mol. Spectrosc. 2001, 208, 209.

(17) Tang, J.; McKellar, A. R. W.; Surin, L. A.; Fourzikov, D. N.; Dumesh, B. S.; Winnewisser, G. J. Mol. Spectrosc. 2002, $214,87$.

(18) Surin, L. A.; Fourzikov, D. N.; Lewen, F.; Dumesh, B. S. Winnewisser, G.; McKellar, A. R. W. J. Mol. Spectrosc. 2003, 222, 93.

(19) Dumesh, B. S.; Panfilov, V. A.; Surin, L. A.; Fourzikov, D. N.; Winnewisser, G. JETP Lett. 2004, 80, 98. Translated from: Dumesh, B. 
S.; Panfilov, V. A.; Surin, L. A.; Fourzikov, D. N.; Winnewisser, G. Pis'ma Zh. Eksp. Teor. Fiz. 2004, 80, 107.

(20) McKellar, A. R. W. J. Chem. Phvs. 2001, 115, 3571.

(21) Surin, L. A.; Fourzikov, D. N.; Dumesh, B. S.; Winnewisser, G.; Tang, Jian, McKellar, A. R. W. J. Mol. Spectrosc. 2004, 223, 132.

(22) Xu, Y.; Jäger, W. J. Chem. Phys. 1999, 111, 5754.

(23) McKellar, A. R. W. J. Mol. Spectrosc. 2004, 226, 190.

(24) Surin, L. A.; Fourzikov, D. N.; Giesen, T. F.; Schlemmer, S.; Winnewisser, G.; Panfilov, V. A.; Dumesh, B. S.; Vissers, G. W. M.; van der Avoird, A. J. Chem. Phvs. 2006, 125, 094304.

(25) Vissers, G. W. M.; Wormer, P. E. S.; van der Avoird, A. Phys. Chem. Chem. Phys. 2003, 5, 4767.
(26) Vissers, G. W. M.; He $\beta$ elmann, A.; Jansen, G.; Wormer, P. E. S. van der Avoird, A. J. Chem. Phys. 2005, 122, 054306.

(27) Surin, L. A.; Potapov, A. V.; Müller, H. S. P.; Panfilov, V. A.; Dumesh, B. S.; Giesen, T. F.; Schlemmer, S. J. Mol. Struct. 2006, 795, 198.

(28) Xia, C.; McKellar, A. R. W.; Xu, Y. J. Chem. Phys. 2000, 113 525 .

(29) Surin, L. A.; Dumesh, B. S.; Lewen, F.; Roth, D. A.; Kostromin, V. P.; Rusin, F. S.; Winnewisser, G.; Pak, I. Rev. Sci. Instrum. 2001, 72, 2535.

(30) McKellar, A. R. W. Private communication, 2005. 